\title{
Interactive comment on "Webcam network and image database for studies of phenological changes of vegetation and snow cover in Finland, image time series from 2014-2016" by Mikko Peltoniemi et al.
}

\section{Anonymous Referee \#2}

Received and published: 30 October 2017

The paper by Peltoniemi et al. presents an extensive network of automated cameras that are installed at field sites across Finland, spanning from the north to the south of the country. This paper is very useful for people who would wish to access this data for studies on the timing of phenological stages of vegetation. Furthermore, the paper includes a useful analysis of the daily variance in the GCC greenness index.

I have few comments other than those already raised by the other reviewer. My main point of criticism would be towards the claim in the abstract that this data can be used as 'ground truths to earth observations'. I agree that the timing of e.g. greening and 
senescence can be derived from this camera network, which may be useful to interpret remote sensing data, but they can't act as a real ground truth. As is clear from Figure 2 , many of the cameras are pointed horizontally towards tree canopies or aimed at the forest floor. Satellites, meanwhile, look straight down and see a completely different aspect of leaves, stems and the understory of the forest. This will lead to especially large differences when trees are partially covered in snow (as is the case at Hyytiälä, today on October 30th), but holds true for other parts of the year as well. Rather than suggesting that this data represents a ground truth to earth observations, I would suggest to say that this data can supplement earth observations.

Otherwise, I second the comment from the other reviewer that the English of this paper needs to be improved. The site descriptions are riddled with grammatical errors, but the rest of the paper could also benefit from a thorough language check.

Other, minor comments:

- Page 4, line 12: This sounds arbitrary. What determines whether an image is taken at full or a quarter resolution? And what is this resolution?

- Page 4, line 12: jpegs are compressed images by definition (that's how this format works). Do you mean the highest quality setting?

- Page 7, line 28: 'weather stations' should be singular, I assume?

- Page 9, lines 8-9: why choose medians? Was the daily pattern in GCC not normally distributed? How does it influence the data if a simple average is chosen?

- Page 9, line 21: I think you mean classes 1 and 2. Class 3 is the only one without a gap.

Interactive comment on Earth Syst. Sci. Data Discuss., https://doi.org/10.5194/essd-2017-62, 2017. 